\title{
Introduction: Semiotic Scaffolding
}

\author{
Jesper Hoffmeyer ${ }^{1}$
}

Received: 4 March 2015 / Accepted: 18 March 2015 / Published online: 2 April 2015

(C) Springer Science+Business Media Dordrecht 2015

\section{Introduction: Semiotic Scaffolding}

A central idea in biosemiotic writings has been the idea of growth in semiotic freedom as a persistent trend in evolution (Hoffmeyer 1992). By semiotic freedom we mean the capacity of species or organisms to derive useful information by help of semiosis or, in other words, by processes of interpretation in the widest (Peircean) sense of this term. While even bacteria have a certain very limited ability to interpret cues in the medium this ability obviously becomes more developed in more complex organisms, and is typically most developed in big-brained animals that are late arrivals at the evolutionary scene. The evolution of a richer semiotic capacity is of course only one among many strategies available in the evolutionary game. Yet, this particular strategy potentially ignites a self-perpetuating evolutionary dynamics, since each step taken by a species along this route potentially opens new agendas for further change:

the more capable some species are of anticipating and interpreting complex and fastchanging situations or events, the more will evolution favor the development in other species of a well-adjusted set of semiotic tools. Note that tools for anticipation, semiotic tool sets, are categorically different from tools for exerting a direct material interaction. Non-semiotic tools such as teeth and claws, muscular power, speed, size etc. are relatively rigidly bound to the nature of the challenge for which they evolved. The peak speed of a cheetah has to match the speed of prey animals, and the anatomy of herbivore teeth has to obey a rigid set of rules pertaining to the chewing of plant material. Now, consider Stuart Kauffman's humorous suggestion of a person surviving an earthquake because the beating pattern of her heart made her anticipate the quake and thus escape it (Kauffman 2000, 130). Here the relevant semiotic tool was a capacity to interpret a significant pattern of heart beats as referring to an upcoming earthquake. The example illustrates the fact that there practically are no compelling rules binding the materiality of the anticipating tool to the materiality of the challenge. The range of

Jesper Hoffmeyer

jhoffmeyer@me.com

1 Biological Institute, University of Copenhagen, Borgergade 42, 5 tv, 1300 Copenhagen K, Denmark 
possibilities for the production of evolutionary novelties is therefore dramatically widened up when anticipatory behavior has become a decisive fitness factor.

A very important aspect of this evolutionary process is the building up of a pattern of semiotic scaffoldings, a tightly wound web of checks and balances gradually establishing itself through myriads of semiotic interactions between organisms: Nature in fact is not so much about "tooth and claw" as it is about sensing, interpreting, coordinating and social co-operation.

Semiotic scaffolding, however, is not only involved in maintenance of balances at the ecological level. As the collection of papers published in this volume illustrates, semiotic scaffolding is a key element in evolutionary processes, whether they are played out at the level of the individual organism, the level of integrated ecological units, or the level of social interactions in human history.

So, what is semiotic scaffolding? Perhaps the shortest way to answer this question is to say, that semiotic scaffolding is what makes history matter to an organism (or a cultural system). Let me illustrate this by means of the simplest system in which semiotic scaffolding operates, that of bacteria. Although bacteria are simple compared to eukaryotic cells, they are nevertheless extremely complex when compared to known inorganic systems at the same scalar level. In the tiny volume of a bacterial cell there are many millions of protein molecules, most of them enzymes catalyzing a diversity of metabolic processes. Obviously a functional coordination of this mess of metabolic processes requires the incessant monitoring of key parameters such as the concentration of distinct metabolites. A measurement necessarily consists in evaluating a given situation with respect to a certain standard, and as such a measurement necessarily also is an interpretation: something is or is not as it "should" be. Here the term "should" points us to the presence in the system, the bacterium, of a kind of value. This value is a systems property, not a property of any singular calibration mechanism operative in the system, and it consists in a complex fine-tuned set of scaffolding mechanisms based on semiosis, sign activity that has become established through the building up in the course of evolution. Just like traffic in a city is scaffolded by myriad signposts visible along the streets or painted upon road surfaces, thus molecular signposts inside the bacterial cell or at the surface of the cell serve to safeguard the organizational functionality of the bacterium. This matrix of interdependent semiotically constrained cellular interactions, whereby the flows of energy and metabolites through the cell are each kept at an optimal level relative to bacterial functionality, is what I understand as semiotic scaffolding at the level of a bacterial cell. Through the combined cytoplasmic and genomic inheritance this elaborate system of semiotic interactions is reproduced in each new cell generation assuring that the evolutionary past lies ingrained in the bacterium as a particular semiotic scaffolding or, as I said: history matters to the cell. And this is exactly the point that distinguishes living systems from non-living systems, the presence in the former of a historically created semiotic interaction mechanism which has no equal in the latter (Hoffmeyer 2007).

All the papers collected in this special issue of Biosemiotics are engaged in studying the operation of semiotic scaffolding mechanisms as they occur at different levels of complexity. Obviously we have no intention of presenting an exhaustive treatment of the many facets of this principle as displayed in nature or in culture. The ambition has been rather to illustrate the unifying capacity of the concept. Sign processes easily transcend hierarchical borders because the material substrate for the entities united in 
the triadic sign process is of no relevance for establishing it in action, in semiosis (Deely 2009). Sign processes painlessly cross the borders between those domains of reality that in the Cartesian understanding are unbridgeable separate orders, science and the humanities, body and mind, nature and culture.

The order of papers in this issue partly reflects a "bottom-up" logic where the semiotic scaffoldings safeguarding functionality at early steps in evolution are discussed in the beginning. In later stages of evolution, however, the multidimensionality of semiotic scaffoldings forbids an ordering after any simple scale, and the ordering of the contributions is more free.

The first major challenge in evolution that called for construction of more sophisticated semiotic scaffoldings than those to be found in bacterial cells must have been the symbiotic integration of a number of prokaryotic cells into one much larger and much more complex eukaryotic cell. Inside this big eukaryotic cell a number of functions that in the bacteria (or Archaea) was located at the cell membrane was now taken over by internal organelles (chloroplasts, mitochondria, etc.) that probably themselves were of bacterial origin (Margulis 1981). Since all eukaryotic cells share the same peculiar internal architecture it is generally assumed that the prokaryoteeukaryote transition has happened only once, or at least that all extant groups of eukaryote forms evolved from one last eukaryote common ancestor (LECA), as has also been shown by genomic analysis (Koonin 2010). At what time this occurred is debatable, but it must have happened at some point between 2 billion years and 1 billion years ago. Life is generally believed to have been present on Earth for 3.8 billion years, so it took evolution 2 billion years or more to invent the eukaryotic cell, which indicates how difficult or even unlikely this step actually was.

The next major step in the evolution of sophisticated semiotic scaffolding systems was the transition from unicellularity to multicellularity discussed in my own contribution to this issue: "Semiotic scaffolding of multicellularity". Once eukaryotic cells were present it took evolution considerably shorter time to produce multicellular life. Metazoan life forms have been around for some 700 million years. Multicellularity has appeared many times in evolution, but only in three cases, that of fungi, plants and animals, has this transformation led to the production of a large quantity of taxonomic groups that include many species. The hard problem at this stage of evolution was not so much to construct multicellularity as such, rather it was to assure that such anomalous individuals consisting of a number of individual totipotent units, cells, could suppress the inherent agency of the individual units and "persuade" them to obey the necessities of cooperation in the interests of the whole.

The birth of multicellularity was also the birth of true individuality in the sense of having a life-history with a distinct beginning, followed by a series of developmental stages leading to adulthood and the reproductive state, and finally senescence followed by death of the individual. The application of the concept of scaffolding in the context of the developing organism is discussed in details in the paper Developmental scaffolding by Franco Giorgi and Luis Bruni. They conceive of the embryo as an integral whole, comprised of several hierarchical modules as in a recurrent circularity of emerging patterns. A wide range of genetic, cellular and morphological mechanisms allows the scaffolding to integrate these modular variations into a functionally coordinated unit. The full hierarchy of a multi-level scaffolding is eventually attained when the embryo acquires the capacity to impose a number of developmental constraints on 
its constituting parts in a top-down direction. The acquisition of this capacity allows a semiotic threshold to emerge between the living cellular world and the underlying nonliving molecular world.

A potential confusion should be addressed in this context. The term scaffolding has appealed to theoreticians inside a wide spectrum of approaches to life and cognition (see also Cobley's and Stjernfelt's paper in this issue). There is however a decisive difference between the ideas of semiotic scaffolding and developmental scaffolding as this concept has recently been introduced (Griesemer 2014). That the scaffold is semiotic implies that it is of a relational nature; it doesn't exist as a material structure the way developmental scaffoldings are normally understood, because the way it operates is through the stabilizing effect of sign action or semiosis that cannot, of course, be described by just enumerating the components of the sign vehicles or referent objects implied. We submit that "semiotic scaffolding" is a necessary addition to the tool set of developmental biology.

One often overlooked feature of multicellularity is that it opens the way for different species to co-inhabit the same body. Multicellular organisms often, perhaps always, host a range of microorganisms. In "The birth of the holobiont: Multi-species birthing through mutual scaffolding and niche construction" Lynn Chio and Scott F. Gilbert discuss the vital reciprocal scaffoldings established through the interaction between host and symbionts in these holobionts, i.e., multicellular eukaryotes with multiple species of persistent symbionts. The human organism is itself a holobiont: about onethird of the metabolites in human blood are derived from bacteria, and in addition to our own 22.000 human genes we also get help from a huge number of genes carried by our many different symbiont species.

In his paper "Scaffolding and mimicry: A semiotic view of the evolutionary dynamics of mimicry systems" Timo Maran uses the example of mimicry to throw light on the ways semiotic scaffolding may operate at the ecological level. Mimicry is a process in which a feature of one organism, the mimic, resembles some feature of another organism, the model, that usually belongs to a different species. This resemblance usually is treacherous and brings us into the world of cheating and deceit, which is one important semiotic aspect of ecological scaffolding.

Kalevi Kull, in "Evolution, choice, and scaffolding: Semiosis is changing its own building", relates the concept of scaffolding to other fundamental semiotic concepts, and analyses their interrelation in the context of evolutionary processes. He sees semiosis as an active meaning-seeking-making process, and observes that it results in the formation of relatively static or even solid structures that somehow in themselves embed the findings of such active searching-event of semiosis. The resulting structure is a scaffolding device. It canalizes further behaviors and frames the formation of new habits.

That life is ultimately and of necessity always cognition and learning in the widest sense of these terms was an essential point in Kalevi Kull's argument. Don Favareau in his paper "Symbols are grounded not in the world, but in the scaffold: How the referential generality of symbol Scaffolding grows minds" takes this insight and expands it into an analysis of how, as Peirce famously claimed, "symbols grow". The point here is that semiotic scaffoldings not only facilitate temporal stability, they also afford "spatial touch points" in the semantic topology of the sign system. Such touch points enable human symbol users to intelligently discuss abstract, counterfactual and 
multiply referential meanings, and by so doing the scaffolding itself, as scaffolding, becomes configured into new scaffoldings thereby facilitating the process whereby "symbols grow".

"Attachment relationships as semiotic scaffolding systems" by Patricia Crittenden and Andrea Landini focuses on parents and infants. The Dynamic-Maturational Model of Attachment and Adaptation (DMM) proposes that (1) exposure to danger organizes both psychological and behavioral protective strategies, (2) the family is the basic organizational unit, (3) attachment figures shape organizational processes, and (4) developmental pathways across the lifespan explain the occurrence of maladaptive functioning. According to the DMM, somatic discomfort, affective distress, and cognitive dissonance, together with failing protective strategies, define psychopathology and dispose for seeking help. The authors suggest that, in psychotherapy, therapists function as transitional attachment figures using semiotic scaffolding processes that mimic parent-infant scaffolding processes, to initiate change with patients.

Claus Emmeche in "Semiotic scaffolding of the social self in reflexivity and friendship" departs from Aristotle's classical idea of the friend as 'another self' and surmises that this might be seen as an early attempt to point to rationality (and its dialogic aspect) as a device for scaffolding the emergence of a mature kind of selfhood. It is shown that although processes of reflexivity and friendship can indeed be seen as instances of semiotic scaffolding of the emerging self, such processes are heterogeneous and contingent upon different modes of reflexivity. Thus semiotic scaffolding should be understood as a multi-level phenomenon.

In "Scaffolding development and the human condition" Paul Cobley and Frederik Stjernfelt discuss what a theory of semiotic scaffolding may offer to an understanding of the humanities' contemporary role. They argue that the essence of being human is not given once and for all but finds expression in the ever-evolving externalizations of symbolic forms. Semiotic scaffoldings are not something that necessarily announce themselves with immediate use-value. They develop, instead, often in a labyrinthine fashion, under the influences within distinct and sometimes clashing social formations. Aesthetic behavior, therefore, is not so much a subsidiary to the ongoing process of survival, but an integral part of human modeling that has enabled humans not only to negotiate the complexity of their environment more ably, but also to envisage new (aesthetic) worlds in a manner which is not identical to, but is cognate with, attempting to anticipate the future.

Wendy Wheeler, in "The wrecked vessel: The effects of gnosticism, nominalism and the protestant reformation in the semiotic scaffolding of modern scientific consciousness", carries the discussion of semiotic scaffoldings into the landscape of the production of science and the science of production. The main tenet of this historical analysis is that science of today retains important traces of gnostic influences which accompanied Christianity from the beginnings. These traces became reinforced or scaffolded through the nominalist persuasions of postreformation thinking and were carried forward to be realized in the modern "megamachine" (Lewis Mumford). The essay suggests that semiotically revivified life sciences might be instrumental in resolving systemic and ecological problems connected to this critical development.

In "Semiotic scaffolding in Mathematics" Mikkel Willum Johansen and Morten Misfeldt investigate if and how cognitive artifacts offer semiotic scaffolding in the field of mathematics and, furthermore, discuss how such scaffolding functions might have 
influenced the development of mathematics. The analyses is focused on three cases, 1) the role played by semiotic scaffoldings in concrete, simple calculations; 2) the use of abstract symbols as scaffolding tools in the development of a mathematical theory; and 3 ) the scaffolding role played by tool/object conversions in the development of a mathematical theory.

The final word in this collection of papers goes to John Deely with the paper "Building a scaffold: Semiosis in nature and culture". Deely defends his extended view of semiosis as being both prior to and independent of living beings. In his own words:

Wherever dyadic interactions result indirectly in a new condition that either moves the universe closer to being able to sustain life, or moves life itself in the direction not merely of sustaining itself but opening the way to new forms of life, we encounter a 'thirdness' in nature of exactly the sort that semiosic triadicity alone can explain. This is the process, both within and without the living world, that requires scaffolding .... Thus, while the universe does not consist exclusively of signs, it is yet perfused by Thirdness as the action of signs, beginning as a 'physiosemiosis', and only culminating much later (as far as we are concerned!) as 'anthroposemiosis'.

\section{References}

Deely, J. (2009). Pars pro toto from culture to nature: an overview of semiotics as a postmodern development, with an anticipation of developments to come. American Journal of Semiotics, 25(1/2), 167-192.

Griesemer, J. R. (2014). Reproduction and the scaffolded development of hybrids. In L. R. Caporael, J. R. Griesemer, \& W. C. Wimsatt (Eds.), Developing scaffolds in evolution, culture, and cognition (pp. 2355). Cambridge: MIT Press.

Hoffmeyer, J. (1992). Some semiotic aspects of the psycho-physical relation: The endo-exosemiotic boundary. In T. A. Sebeok \& J. Umiker-Sebeok (Eds.), The semiotic web 1991: Biosemiotics (pp. 101-123). Berlin/ New York: Mouton de Gruyter.

Hoffmeyer, J. (2007). Semiotic scaffolding of living systems. In M. Barbieri (Ed.), Introduction to biosemiotics (pp. 149-166). Dordrecht: Springer.

Kauffman, S. (2000). Investigations. Oxford: Oxford University Press.

Koonin, E. V. (2010). The origin and early evolution of eukaryotes in the light of phylogenomics. Genome Biology, 11, 209. doi:10.1186/gb-2010-11-5-209.

Margulis, L. (1981). Symbiosis in cell evolution: Life and its environment on earth. San Fransisco: Freeman. 